American Journal of Applied Science 2 (11): 1491-1492, 2005

ISSN 1546-9239

(c) 2005 Science Publications

\title{
On Permutable Subgroups of n-ary Groups
}

\author{
Awni Fayez Al-Dababseh \\ Department of Mathematics and Statistics, Al-Hussein Bin Talal University, Ma'an, Jordan
}

Abstract: It is proved that every permutable subgroup of a finite n-ary group is subnormal.

Key words: finite n-ary group, permutable n-ary group, subnormal n-ary group

\section{INTRODUCTION}

We remind that, the system $\mathrm{G}=\langle\mathrm{X},()\rangle$ with one $\mathrm{n}$-ary operation ( ) is called $\mathrm{n}$-ary group ${ }^{[1,2]}$, if it is associative and every one of the equations.

$\left(a_{1} a_{2} \ldots . a_{i-1} \times a_{i+1} \ldots \ldots a_{n}\right)=a$

is solvable in $X$, where $a_{1}, \ldots a_{n}, a \in X, i=1,2, \ldots, n$. Throughout this study all n-ary groups are finite. Let $\mathrm{G}$ be $\mathrm{n}$-ary group and let $\mathrm{H}$ is a subgroup of $\mathrm{G}$, then $\mathrm{H}$ is called permutable n-ary group if $\mathrm{HT}=\mathrm{TH}$ for all subgroups $\mathrm{T}$ of $\mathrm{G}$.

It is known however, that every permutable subgroup of a finite group is subnormal ${ }^{[3,4]}$. In this study we prove this property for $\mathrm{n}$-ary groups.

\section{PRELIMINARIES}

Notation is standard ${ }^{[2]}$

$X_{m}^{k}$-the sequence $X_{m} X_{m+1} \ldots X_{k}$ (if $\mathrm{m}=\mathrm{k}$ then $\left.X_{m}^{m}=x_{m}\right)$.

Definition 1: Let $\mathrm{G}$ be $\mathrm{n}$-ary group, then $x_{1}^{k(n-1)}$ is an identity if $\left(x x_{1}^{k(n-1)}\right)=\left(x_{1}^{k(n-1)} x\right)=x$ for all $\mathrm{x} \in \mathrm{G}$.

Definition 2: Let $\mathrm{G}$ be $\mathrm{n}$-ary group and let $\mathrm{x} \in \mathrm{G}$, then the sequence of elements $\bar{x}$ of $\mathrm{G}$ is called an inverse of $\mathrm{x}$ if $x \bar{x}$ is an identity.

Let $\mathrm{H} \leq \mathrm{G}$ and $x_{1}^{i}, y_{1}^{j}$ are sequences of elements of $\mathrm{G}$, where $\mathrm{i}+\mathrm{j}=\mathrm{k}(\mathrm{n}-1)[\mathrm{k} \in \mathrm{N}]$, then the symbol $\left[X_{1}^{i} H y_{1}^{j}\right]$ denote all elements $\left(x_{1}^{i} h y_{1}^{j}\right)$ where $\mathrm{h} \in \mathrm{H}$.

By analogous of binary groups n-ary subgroup $\mathrm{H}$ of a group $\mathrm{G}$ is called normal if for any $\mathrm{x} \in \mathrm{G}$ and for any sequence $\bar{x}$ we have $x H \bar{x}=H$.

Definition 3: $\mathrm{N}$-ary subgroup $\mathrm{H}$ of a group $\mathrm{G}$ is called subnormal in $\mathrm{G}$ if:

$H=N_{0} \leq N_{i} \leq \ldots \leq N_{t-1} \leq N_{t}=G$

Where $\mathrm{N}_{\mathrm{i}}$ is a normal in $\mathrm{N}_{\mathrm{i}+1}, \mathrm{i}=0,1, \ldots, \mathrm{t}-1$.

If $\mathrm{H}$ and $\mathrm{T}$ are subgroups of $\mathrm{n}$-ary group $\mathrm{G}$, then $\left[\begin{array}{c}i \\ H T\end{array}\right]$ is the set of all products $\left(h_{1} . . h_{i} t_{1} t_{n-i}\right)$, where $\mathrm{h}_{\mathrm{i}}$ $\in \mathrm{H}$ and $\mathrm{t}_{\mathrm{j}} \in \mathrm{T}$.
Lemma $\mathbf{1}^{[2]}$ : Let $\mathrm{H}$ and $\mathrm{T}$ are subgroups of $\mathrm{n}$-ary group G such that

$\left[\begin{array}{c}n-1 \\ T\end{array}\right]=[\stackrel{n-1}{T} H]$, then $\mathrm{B}=[H \stackrel{n-1}{T}]$ is a subgroup of $\mathrm{G}$ and $B \supseteq H$.

Subgroup $\mathrm{H}$ of $\mathrm{n}$-ary group $\mathrm{G}$ is called permutable if for any subgroup $\mathrm{T}$ from $\mathrm{G}$ we have

$T \cap H \neq \Phi$ and $[H \stackrel{n-1}{T}]=[\stackrel{n-1}{T} H]$

Lemma $2^{[2]}$ : Let $\mathrm{H}$ and $\mathrm{T}$ are subgroups of n-ary group

$\mathrm{G}$. If $\mathrm{H} \cap \mathrm{T} \neq \phi$, then

$\left|\left[H \frac{n-1}{H} T\right]\right|=\frac{|H||T|}{|H \cap T|}$

\section{MAIN RESULTS}

We are now to prove the following.

Lemma 3: If $\mathrm{H} \mathrm{u} \mathrm{T}$ are permutable subgroups of n-ary group $\mathrm{G}$, then $[H \stackrel{n-1}{T}]$ is permutable subgroup of $\mathrm{G}$.

Proof: Let D any subgroup of $n$-ary group $G$, then by the definition of permutable subgroup $\mathrm{H} \cap \mathrm{D} \neq \phi$. By lemma $1 \mathrm{H} \leq[H \stackrel{n-1}{T}]$ and it is mean that $\mathrm{H} \cap \mathrm{D} \subseteq$ $[H \stackrel{n-1}{T}] \cap \mathrm{D} \neq \phi$.

Now since

$\left[\left[\begin{array}{ll}H & T-1\end{array}\right]^{n-1} D\right]=\left[H \stackrel{n-2}{T} T D^{n-1}\right]=\left[\begin{array}{cc}H & n-2 n-1 \\ T & D\end{array}\right]=$

.... $[H \stackrel{n-1 n-1}{D} T]=\left[\stackrel{n-1}{D} H^{n} \stackrel{n-1}{T}\right]=[\stackrel{n-1}{D}[H \stackrel{n-1}{T}]]$

So $[H \stackrel{n-1}{T}]$ is permutable subgroup in $\mathrm{G}$.

Lemma 4: Let $\mathrm{H}$ be a subgroup of n-ary group $\mathrm{G}$. Then if for some element $\mathrm{x} \in \mathrm{G}$ and for some sequence of inverse $(\bar{x})$ of $\mathrm{x}$ we have $\left[H H_{1}^{n-1}\right]=\mathrm{G}$, where $H_{1}=x H \bar{x}$, then $\mathrm{H}=\mathrm{H}_{1}$.

Corresponding Author: Awni Fayez Al-Dababseh, Department of Mathematics and Statistics, Al-Hussein Bin Talal University, Ma'an, Jordan 


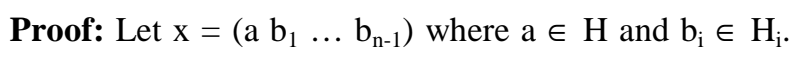
Let $\bar{b}_{i}$ be a sequence of elements from $\mathrm{H}_{1}$ which are inverses for $b_{i}, i=1,2, \ldots, n-1$. Then

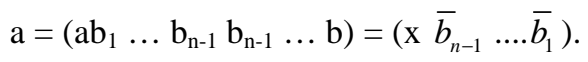

It is clear, that $b_{1} \ldots b_{n-1} x$ is the sequence of inverses for $a$. That means if $\bar{a}$ is any sequence of elements of $\mathrm{H}$ that inverse for $\mathrm{a}$, then $\mathrm{H}=[a H \bar{a}]=$ $\left[\left(x \bar{b}_{n-1} \ldots b_{1}\right) H\left(b_{1} \ldots b_{n-1} \bar{x}\right)\right]=[x H \bar{x}]=H_{1}$.

Lemma 5: Let $\mathrm{x}$ be an element of $\mathrm{n}$-ary group $\mathrm{G}$ and let $\varphi_{x}: \mathrm{G} \rightarrow \mathrm{G}$ a map defined by $\varphi_{x}(g)=x g \bar{x}$ where $\mathrm{g} \in$ $\mathrm{G}$ and $\bar{x}$ is some sequence that is inverse for $\mathrm{x}$. Then $\varphi_{x}$ is an automorphism of G.

Proof: For any sequence of element $g_{1}^{n}$ from $\mathrm{G}$ we have

$$
\begin{aligned}
& \varphi_{x}\left(g_{1} \ldots g_{n}\right)=x\left(g_{1} \ldots g_{n}\right) \bar{x}= \\
& \left(x\left(g_{1} \bar{x} x\right)\left(g_{2} \bar{x} x\right) \ldots .\left(g_{n-1} \bar{x} x\right) g_{n} \bar{x}\right)= \\
& \left(\left(x g_{1} \bar{x}\right)\right)\left(x g_{2} \bar{x}\right) \ldots . .\left(x g_{n} \bar{x}\right)=\left(g_{1}^{\varphi x} g_{2}^{\varphi x} \ldots . g_{n}^{\varphi x}\right)
\end{aligned}
$$

So $\varphi_{x}$ is an endomorphism of $\mathrm{n}$-ary group $\mathrm{G}$.

If $\mathrm{g} \in \mathrm{G}$, then $\varphi_{x}(\bar{x} g x)=(\mathrm{x}(\bar{x} g x) \bar{x})=\mathrm{g}$. It means that $\ell \mathrm{x}$ is an epimorphism. It is obvious that $\varphi_{x}$ is an injection.

Theorem: If $\mathrm{H}$ is a subgroup of $\mathrm{n}$-ary group $\mathrm{G}$ that is permutable with any subgroup of $G$, then $H$ is a subnormal in $\mathrm{G}$.

Proof: We prove by induction on the order of n-ary group $\mathrm{G}$. let $\mathrm{N}$ is the greatest permutable subgroup of $\mathrm{G}$ $(\mathrm{N} \neq \mathrm{G})$ that contains the subgroup $\mathrm{H}$.
We show that $\mathrm{N}$ is a normal subgroup of $\mathrm{G}$. let $\mathrm{N}$ is not normal subgroup. By the definition of normal subgroup we can find some $\mathrm{x} \in \mathrm{G}$ such that $\mathrm{xN} \bar{x} \neq \mathrm{N}$ where Error! Bookmark not defined. is some sequence that is inverse of $\mathrm{x}$. let $\varphi_{x}: \mathrm{G} \rightarrow \mathrm{G}$ defined by $\varphi_{x}(\mathrm{~g})=\mathrm{xg} \bar{x}$ for all $\mathrm{g} \in \mathrm{G}$. by lemma $5, \varphi_{x}$-is an automorphism n-ary group $\mathrm{G}$. That means $\mathrm{xN} \bar{x}$ is a permutable subgroup of n-ary group $\mathrm{G} \mathrm{u}|N|=|x N \bar{x}|$. Applying lemma 1 we have $D=\left[\begin{array}{l}n-1 \\ N\end{array}\right]=[N \stackrel{n-1}{N}]$ which contains $\mathrm{N}$ subgroup $\mathrm{n}$-ary group $\mathrm{G}$, where $N_{1}=x N \bar{x}$. According to lemma 2 the order of this subgroup is:

$d=\left|N_{1}^{n-1} N\right|=\frac{\left|N_{1}\right||N|}{\left|N_{1} \cap N\right|}$

Since $\mathrm{N} \neq x N \sqrt{x}$ and $|N|=|x N \sqrt{x}|$, then $\mathrm{d}>\mathrm{N}$. But by Lemma 3 subgroup D is permutable in G. That means $\mathrm{D}=\mathrm{G}$ and this contradict lemma 4 . So $\mathrm{N}$ is a normal subgroup of g. Since $|N|<|G|$ and $\mathrm{H}$ is permutable subgroup of $\mathrm{N}$, then by, choosing group $\mathrm{G}$ we can conclude that $\mathrm{H}$ is subnormal subgroup in $\mathrm{N}$. It means $\mathrm{H}$ is a subnormal subgroup of $\mathrm{G}$.

\section{REFERENCES}

1. Kurash, A.E., 1974. General Algebra (Lectures 1969-70). Nauka, Moscow.

2. Rusakov, C.A., 1992. Algebraic n-ary systems, MN. Nauka and Technika.

3. Stonehewer, S.E., 1972. Permutable Subgroup of Finite Groups. Math Z., 125: 1-16.

4. Schmidt, R., 1994. Subgroup Lattices of Groups. Walter de Gruyter, Berlin-New York. 